\title{
DESENVOLVIMENTO DE SOFTWARES EM PROGRAMAS BRASILEIROS DE PÓS-GRADUAÇÃO EM ENFERMAGEM: PESQUISA DOCUMENTAL
}

\author{
Stephanie Barbosa de Medeiros \\ Mestranda do Programa de Pós-Graduação em Enfermagem, Departamento de \\ Enfermagem, Universidade Federal do Rio Grande do Norte (UFRN). Bolsista do \\ Conselho Nacional de Desenvolvimento Cientifico e Tecnológico (CNPq). Membro do \\ Grupo de Pesquisa: Laboratório de Investigação do Cuidado, Segurança, Tecnologias em \\ Saúde e Enfermagem - UFRN.stephanie_natal@yahoo.com.br \\ Camila Dannyelle Fernandes Dutra Pereira \\ Mestranda do Programa de Pós-Graduação em Enfermagem, Departamento de \\ Enfermagem, Universidade Federal do Rio Grande do Norte (UFRN). Bolsista da \\ Coordenação de Aperfeiçoamento de Pessoal de Nível Superior (CAPES). Membro do \\ Grupo de Pesquisa: Laboratório de Investigação do Cuidado, Segurança, Tecnologias em \\ Saúde e Enfermagem - UFRN. camilafernandes_enf@hotmail.com \\ Yole Matias Silveira de Assis \\ Acadêmica de Enfermagem da Universidade Federal do Rio Grande do Norte (UFRN). \\ Bolsista de Iniciação Científica PROPESQ/UFRN. Membro do Grupo de Pesquisa: \\ Laboratório de Investigação do Cuidado, Segurança, Tecnologias em Saúde e Enfermagem \\ - UFRN.yole_matias@,hotmail.com \\ Francis Solange Vieira Tourinho \\ Doutora, Professora Adjunto do Programa de Pós-Graduação em Enfermagem, \\ Departamento de Enfermagem, Universidade Federal do Rio Grande do Norte (UFRN). \\ Membro do Laboratório de Inovação Tecnológica em Saúde - (LAIS)/HUOL/UFRN. \\ Líder do Grupo de Pesquisa: Laboratório de Investigação do Cuidado, Segurança, \\ Tecnologias em Saúde e Enfermagem - UFRN. francistourinho@gmail.com \\ Viviane Euzébia Pereira Santos \\ Doutora, Professora Adjunto do Programa de Pós-Graduação em Enfermagem, \\ Departamento de Enfermagem da Universidade Federal do Rio Grande do Norte (UFRN). \\ Vice-líder do Grupo de Pesquisa: Laboratório de Investigação do Cuidado, Segurança, \\ Tecnologias em Saúde e Enfermagem - UFRN. vivianeepsantos@gmail.com
}

\section{RESUMO}

Objetivo: Caracterizar as dissertações e teses disponíveis no Banco de Teses da Coordenação de Aperfeiçoamento de Pessoal de Nível Superior que versam sobre o desenvolvimento de software para a área da saúde e enfermagem. Método: Pesquisa documental realizada através de um protocolo estruturado com os indicadores: nível acadêmico; local do estudo; ano de publicação; subárea do conhecimento; desenho metodológico; temática abordada. Resultados e Discussão: Foram encontrados 37 estudos, entre teses e dissertações, e a partir dos critérios de inclusão foram selecionados oito estudos. O perfil dos estudos demonstrou que $62,5 \%$ são referentes ao mestrado; a maioria foi publicada no ano de 2009; 62,5\% desenvolvidos no estado de São Paulo; $50 \%$ fez referencia ao termo geral "enfermagem"; $25 \%$ são pesquisas metodológicas; e $50 \%$ tratam da criação de sistemas informatizados utilizados como metodologias de ensino. Conclusão: A pós-graduação em enfermagem no país está buscando desenvolver mais estudos 
relacionados à temática abordada, verificando-se também a necessidade de se avaliar futuramente as publicações em periódicos provenientes dos estudos analisados.

PALAVRAS-CHAVE: Enfermagem, Software, Tecnologia.

\title{
SOFTWARE DEVELOPMENT IN BRAZILIAN NURSING GRADUATE PROGRAMS: DOCUMENTARY RESEARCH
}

\begin{abstract}
Objective: To characterize the dissertations and theses available on Theses Database of Coordination for the Improvement of Higher Education Personnel that deal with software development for healthcare and nursing. Method: Documentary research carried out through a structured protocol with the indicators: academic level; place of study; year of publication; subarea of knowledge; methodological design; and theme. Results and Discussion: 37 studies were found including theses and dissertations, and based on the inclusion criteria we selected eight studies. The profile of the studies showed that $62.5 \%$ are from masters courses; most were published in 2009; 62.5\% developed in the state of São Paulo; $50 \%$ referred to the general term "nursing"; $25 \%$ are methodological researches; and $50 \%$ deal with the design of computerized systems used as teaching methodologies. Conclusion: Nursing graduate in Brazil is seeking to develop more studies related to the topic addressed, and we also verify the need to assess in the future the publications in the journals from the studies analyzed.
\end{abstract}

KEYWORDS: Nursing, Software, Technology.

\section{DESENVOLVIMENTO DE SOFTWARES EM PROGRAMAS BRASILEIROS DE PÓS-GRADUAÇÃO EM ENFERMAGEM: PESQUISA DOCUMENTAL}

\section{INTRODUÇÃO}

Os avanços tecnológicos ocasionaram mudanças em várias áreas da contemporaneidade contribuindo para o desenvolvimento de atividades realizadas por diferentes profissionais. Essas inovações, avanços e descobertas geraram profundas modificações na sociedade, no trabalho, na educação e na saúde, caracterizando-se por acontecerem de forma rápida e frequente [1,2].

No campo da saúde, percebe-se um processo acelerado de transformação e inovação tecnológica nas últimas décadas, tanto em nível nacional como internacional, ocasionando mudanças consideráveis na prestação de cuidados [3,4]. A informática em saúde utiliza a tecnologia, como os softwares e hardwares, articulada a conceitos e formas de administrar informações, subsidiando, assim, a assistência em saúde [5].

A literatura refere que os softwares utilizados para a prestação de cuidados aos clientes funcionam como "sistemas de apoio inteligentes nas decisões clínicas, permitindo melhor organização, comunicação, retroalimentação e uma base de análise administrativa" [5]. 
$\mathrm{Na}$ enfermagem, a informática foi sendo estabelecida inicialmente por volta de 1985 [5]. No entanto, estudos desenvolvidos nos Estados Unidos, das décadas de 1960 à 1980, já constatavam a possibilidade dos computadores auxiliarem positivamente a prática da enfermagem [4].

Nos dias hodiernos, é fundamental o reconhecimento da informática como uma relevante ciência que está cada vez mais inserida no processo de trabalho da enfermagem, proporcionando maior qualidade na prática e assistência prestada aos clientes [6,5]. O crescimento do uso de tecnologias, mais claramente nos últimos anos, demonstra que estas consistem em uma metodologia facilitadora para a profissão de enfermagem, subsidiando suas ações [7].

Sua aplicabilidade resulta na melhoria da racionalidade organizacional, uma vez que torna viável e dinâmico o planejamento das ações do enfermeiro, fornecendo-lhe alternativas possíveis de se empregar seus conhecimentos técnico-científicos no âmbito da assistência em enfermagem [4]. Contribui, portanto, nas condições de empoderamento e autonomia dos profissionais em todos os seus contextos de atuação, seja na assistência, gerência ou ensino [6].

Diante da crescente evolução tecnológica na área da saúde, tornou-se possível a participação da enfermagem no desenvolvimento de novas tecnologias, instrumentos importantes na realização da práxis do enfermeiro [5].

Frente a esta conjuntura e considerando a importância de se evidenciarem as tendências das pesquisas no Brasil sobre o desenvolvimento de softwares voltados para a área da saúde, foi realizado um perfil do que vem sendo produzido nos últimos anos no âmbito dos programas de pós-graduação em Enfermagem acerca da temática. Foram elaboradas as seguintes questões norteadoras: 1) Quais as características das dissertações e teses disponíveis no Banco de Teses da Coordenação de Aperfeiçoamento de Pessoal de Nível Superior (CAPES) que versam sobre 'Desenvolvimento de software para a área da saúde e enfermagem'? e 2) Quais as temáticas referentes ao desenvolvimento de software para área da saúde e enfermagem vêm sendo abordadas?

Visando responder a estes questionamentos, o presente estudo tem por objetivo caracterizar as dissertações e teses disponíveis no Banco de Teses da CAPES que versam sobre o desenvolvimento de software para saúde e da enfermagem.

\section{MÉTODO}

Para alcançar o objetivo proposto, o levantamento dos dados foi realizado por meio da pesquisa documental, a qual pode ser uma forma valiosa de abordagem para descobrir aspectos novos de um tema como também complementar informações [8].

O estudo documental compreende as fases, a saber: escolha do tema, delimitação dos objetivos, elaboração do plano de trabalho, identificação e localização das fontes a serem pesquisadas, obtenção e leitura do material identificado, apontamento deste material por meio de fichas, análise, interpretação dos dados e redação final do estudo. Essas fases ocorrem numa sequência natural e de forma articulada [9]. 
Optou-se pela utilização de dissertações e teses, as quais são consideradas fontes importantes para pesquisa, uma vez que são relatórios científicos de pesquisas originais ou relevantes revisões bibliográficas [9]. A escolha deveu-se também, por estes estudos apresentarem alto rigor metodológico e possibilidade de progresso na área científica [10].

Nesse sentido, a pesquisa documental foi desenvolvida no Portal de Periódicos da CAPES, especificamente, no Banco de Teses da CAPES. Criado em 2001, este banco funciona como uma ferramenta de busca e consulta que facilita o acesso às informações de dissertações e teses defendidas, desde 1987, em todo o país [11].

Inicialmente, foi elaborado um protocolo estruturado para o desenvolvimento do estudo, validado por duas das autoras com doutoramento. O protocolo da pesquisa era composto pelas seguintes etapas: objetivo; questões norteadoras; estratégias de busca (banco de dados e descritores controlados); seleção dos estudos (critérios de inclusão e critérios de exclusão); estratégia para coleta de dados dos estudos; estratégia para avaliação crítica do estudo; síntese dos dados.

A coleta dos dados foi realizada aos pares, no mês de julho de 2012. Utilizou-se os descritores "Enfermagem", "Software", "Tecnologia", controlados pelos Descritores de Ciências da Saúde (DEcS), no campo de busca "assunto", por meio da opção "todas as palavras".

A seleção dos estudos baseou-se em critérios previamente elaborados: 1) Critérios de inclusão: dissertações e teses disponíveis eletronicamente no Banco de Teses da CAPES e que abordem acerca do desenvolvimento de software para a área da saúde e suas contribuições para a enfermagem, seja no âmbito do ensino, pesquisa e/ou assistência. 2) Critérios de exclusão: dissertações e teses que não estavam disponíveis eletronicamente na íntegra; que não abordem a temática relevante para o alcance da pesquisa; dissertações e teses duplicadas e estudos que dissertem sobre pesquisas realizadas em animais.

Um instrumento padronizado foi construído para avaliação sistemática de cada estudo selecionado de acordo com os seguintes indicadores da coleta de dados: nível acadêmico (mestrado ou doutorado); local de desenvolvimento do estudo; ano de publicação; subárea do conhecimento; desenho metodológico; temática abordada.

Objetivando uma clara apresentação e compreensão do indicador "temática abordada" dos estudos, foram atribuídos conceitos aos elementos temáticos: 1) Temática "Ensino": estudos acerca do desenvolvimento de tecnologias voltadas para o ensino; 2) Temática "Assistência em saúde": estudos relacionados a criação e aplicação de tecnologias que contribuam para a assistência em saúde e enfermagem; 3) Temática "Avaliação": estudos relacionados a avaliação do desenvolvimento de tecnologias utilizadas na assistência em saúde e enfermagem.

O indicador "desenho metodológico" das teses e dissertações selecionadas foi analisado por meio da caracterização das pesquisas, segundo: objetivos (pesquisa exploratória e descritiva), procedimentos de coleta de dados (pesquisas documentais, experimentais, estudos de caso e metodológicas) e fontes de informação (pesquisas bibliográficas) [12].

Os resultados encontrados e a caracterização dos estudos selecionados foram apresentados por meio de figuras que facilitam a visualização dos dados finais. 


\section{RESULTADOS}

O desenvolvimento da pesquisa se deu a partir de um protocolo previamente estabelecido, através do qual foi possível selecionar 37 estudos, entre teses e dissertações, de acordo com os descritores utilizados. Em seguida, a partir dos critérios de inclusão determinados, a amostra total foi reduzida a um número de oito estudos.

Em relação ao nível acadêmico de tais estudos, cinco (62,5\%) são referentes ao mestrado, dois $(25 \%)$ ao doutorado, e um (12,5\%) ao mestrado profissional. Foi observado também que as pesquisas encontradas foram publicadas entre os anos de 2003 e 2010.

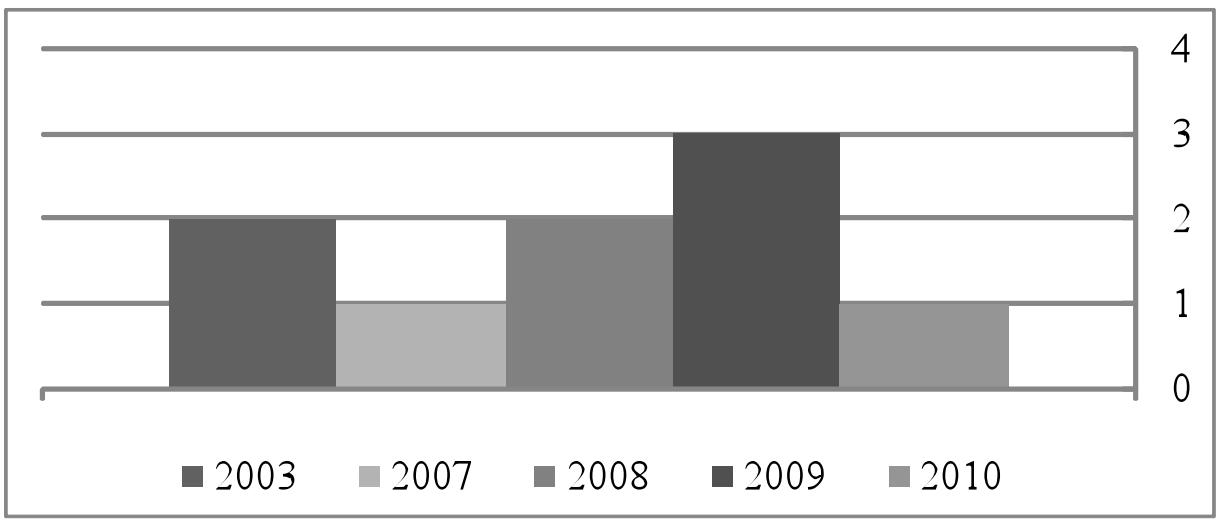

Figura 1 - Distribuição dos estudos de acordo com o ano de publicação. 2012, Natal/RN.

Quanto aos estados onde se localizam as universidades referenciadas pelos estudos, a maior parte destes foi desenvolvida em diferentes instituições no estado de São Paulo (cinco estudos - 62,5\%), dois são provenientes do Rio Grande do Sul (25\%), ficando, portanto, o estado de Santa Catarina com um estudo (12,5\%).

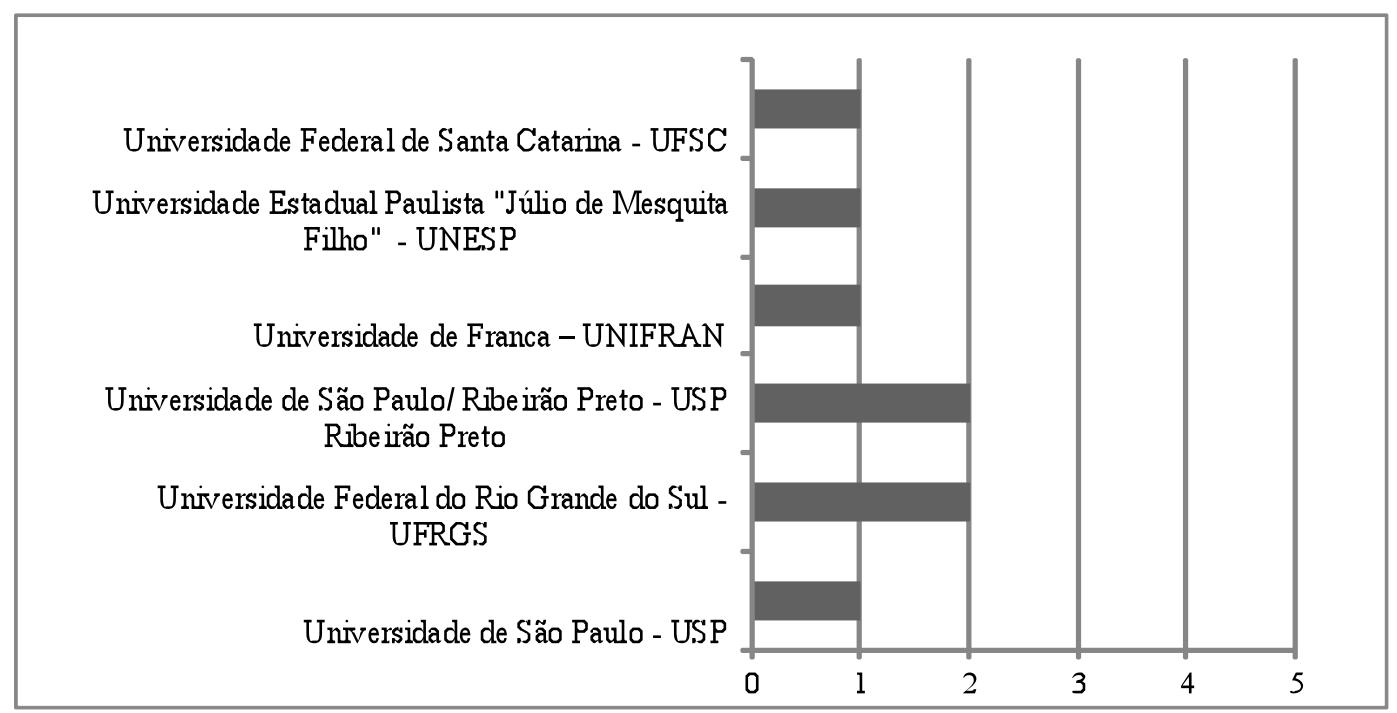

Figura 2 - Distribuição das dissertações e teses de acordo com a instituição de desenvolvimento dos estudos selecionados. 2012, Natal/RN. 
Todas as dissertações e teses selecionadas foram produzidas no âmbito dos programas de pós-graduação em enfermagem do país, destacando-se algumas subáreas, tais como: tecnologia educacional e enfermagem (um - 12,5\%), enfermagem e ciências da saúde (um - 12,5\%), enfermagem e educação à distância (um - 12,5\%) e enfermagem e informática aplicada à saúde (um - 12,5\%). Nos demais estudos foram referidos apenas o termo geral "enfermagem" (quatro - 50\%). Deve-se salientar que foram referidos mais de um tipo de subárea em um único relatório de estudo.

Em relação ao desenho metodológico, os estudos apresentaram os seguintes desenhos: pesquisas metodológicas (25\%); estudo de caso (12,5\%); estudo de caso e pesquisa documental $(12,5 \%)$; pesquisa exploratória e descritiva $(12,5 \%)$; pesquisa descritiva e exploratória $(12,5 \%)$; pesquisa bibliográfica $(12,5 \%)$; pesquisa exploratória $(12,5 \%)$; pesquisa exploratória bibliográfica $(12,5 \%)$.

Após análise da temática abordada nos estudos selecionados, verificou-se que quatro deles $(50 \%)$ tratam da criação de sistemas informatizados utilizados como metodologias de ensino sobre as diversas técnicas da enfermagem, e direcionados para alunos em formação na área. Três estudos (37,5\%) remetem à temática de softwares desenvolvidos especificamente para os profissionais de enfermagem com atuação na prática assistencial, de modo a auxiliar em suas diversas atuações. Por fim, um (12,5\%) estudo aborda sobre a avaliação do uso de tecnologias aplicadas na assistência à saúde.

\section{DISCUSSÃO}

Em nosso país vem ocorrendo um grande crescimento tanto no âmbito da pesquisa quanto no da pós-graduação. Estudos referem uma síntese das perspectivas da expansão da pósgraduação na área da saúde no Brasil, em que foi apontado para os próximos 10 anos um potencial de crescimento de 50\%, com ênfase em alguns cursos desta área, dentre eles o da enfermagem [13].

A pesquisa no Brasil foi delineada a partir do Parecer Maciel-Sucupira (1965), $\mathrm{n}^{\mathrm{o}}$ 977/1965, do Conselho Federal de Educação, formulado por Newton Sucupira, influenciando a formação de uma matriz que gere a produção de conhecimento sustentada, principalmente, no âmbito da pós-graduação das instituições públicas. Assim, este parecer determinou a estruturação dos programas de pós-graduação stricto sensu nas universidades públicas no início da década de setenta [14].

Cabe salientar que a desenvoltura do Brasil na produção de conhecimentos merece destaque no campo internacional, uma vez que apresentou, na última década, um aumento no número de publicações maior que $100 \%$, o que concedeu ao país a nona posição na classificação no mundo. No entanto, essa elevação no número de publicações nacionais e avanço em direção aos grandes produtores mundiais da Ciência, não representa, em termos quantitativos, o esperado ao se considerar o tamanho da população brasileira e seu Produto Interno Bruto (PIB) [14].

Remetendo-se ao contexto da enfermagem, acredita-se que trabalhar a temática proposta possibilita visualizar que a enfermagem vem produzindo na área tecnológica, de forma a contribuir para a realização do processo de trabalho do enfermeiro e de sua equipe. Porém, o desenvolvimento de sistemas tecnológicos exige certo nível de capacitação por parte da enfermagem, tanto na sua área, como no ramo de tecnologias da informação [6]. Desta 
forma, este fato dificulta a aplicação destes sistemas na prática, explicando a pequena quantidade de estudos encontrados durante esta pesquisa.

Em relação ao indicador "nível acadêmico" das pesquisas selecionadas para análise neste estudo, constatou-se um maior número de dissertações em relação às teses, observando-se, assim, similaridade com a realidade da pós-graduação brasileira. No país, o mestrado acadêmico, com 33 mil titulados no ano de 2008, é o tipo de curso de pós-graduação que mais titula e mais cresce. No que concerne ao mestrado profissional, ainda são incipientes, formando em torno de três mil pessoas por ano [15].

No que se refere à temporalidade dos relatórios avaliados nesta pesquisa, pode-se aferir que estes foram publicados nos últimos 10 anos, o que condiz com o cenário de crescimento da pós-graduação brasileira e avanço da área tecnológica evidenciados na literatura.

A saúde é o ramo que mais desenvolve pesquisa científica e tecnológica no Brasil, a qual se concentra mais nos estados da região Sudeste [16]. Esta realidade é consonante com os resultados obtidos nesta pesquisa acerca dos estados de desenvolvimento das dissertações e teses, onde a grande porcentagem dos estudos selecionados é proveniente das universidades do estado de São Paulo.

O setor da pesquisa é, nos dias de hoje, autor da maior parcela das produções da Ciência nas universidades e institutos públicos do Brasil, chegando a atingir o percentual de $90 \%$, emergindo predominante, das regiões Sul e Sudeste [14].

A enfermagem vem vivenciando um processo de evolução, o que vai de encontro ao fato de esta enquanto ciência tem o dever de estar em constante atualização de conhecimentos para que se alcance uma assistência de qualidade. Assim, pesquisas em várias subáreas da enfermagem vêm sendo necessárias, o que se pode comprovar neste estudo, no qual diversas foram abordadas. Vale salientar ainda, que todas essas subáreas estão associadas ao desenvolvimento tecnológico, contribuindo, portanto, para a geração de novos conhecimentos ainda estranhos a muitos profissionais [17].

Quanto ao indicador "desenho metodológico" dos relatórios de pesquisa analisados, não foi identificado estudos do tipo experimentais, os quais possuem o maior nível de evidência no campo científico. Houve destaque para os estudos com desenho de pesquisa metodológica, a qual admite investigações objetivando indicar tecnologias para o desenvolvimento de práticas [12].

Dentre as temáticas abordadas nas teses e dissertações selecionadas, a que teve prevalência nos estudos foi àquela referente às tecnologias com fins educativos na formação dos enfermeiros. Sua relevância se dá pelo estímulo ao aprendizado com aprimoramento da educação, através do uso de mídias digitais, construção de softwares, e outros recursos tecnológicos que complementam a formação profissional [18].

Além desta, foi constatada outra temática em menor quantidade, porém não menos importante, relacionada aos meios eletrônicos criados para aplicação na assistência do enfermeiro. Estas tecnologias são fundamentais para sistematizar o serviço da equipe de enfermagem, já que auxilia no manejo das informações, melhora a eficácia da produtividade, e gera organização do processo de trabalho do enfermeiro [19]. 
O fato de se ter encontrado apenas um estudo relatando sobre o processo de avaliação dos sistemas de informática aplicados na assistência, demonstra a carência de pesquisas voltadas para este ramo, o qual assim como as outras temáticas, tem sua relevância, pois não basta apenas implementar determinada tecnologia, deve-se avaliar a mesma no intuito de observar sua eficiência [17].

Outro ponto que demonstra a importância desta avaliação seria a necessidade de observar a capacitação dos profissionais que utilizam tal tecnologia, fator indispensável para o bom desempenho das funções, visto que o não conhecimento do produto interfere no processo de trabalho, além de gerar o seu desuso e a evasão dos enfermeiros [20].

Em suma, sabe-se que a produção tecnológica da enfermagem está voltada tanto para o cuidado prestado ao paciente, quanto para facilitar o trabalho da sua equipe. Porém, cabe aos profissionais não permitir que o uso deste artifício impeça a prática de um cuidado humanizado ao paciente, lembrando sempre de sua individualidade e o seu bem-estar, e atrelando as tecnologias à comunicação [21].

\section{CONCLUSÃO}

Compreende-se que a pós-graduação de enfermagem no país está buscando desenvolver mais estudos relacionados à temática abordada, posto que cada vez mais os enfermeiros estão expostos as tecnologias e estas fornecem aos profissionais subsídios e possibilidades de aplicação de seus conhecimentos técnico-científicos na assistência. Esse contexto contribui para o fortalecimento do empoderamento do enfermeiro, o qual utiliza deste poder para alcançar seus objetivos, como também se relacionar com a equipe de saúde e população.

De forma sucinta, as dissertações e teses selecionadas no Banco de Teses da CAPES caracterizam-se por serem estudos recentes, na sua maioria dissertações advindas do mestrado acadêmico, oriundas de instituições publicas localizadas na região sudeste, tratando-se predominante da enfermagem com abordagem geral e temática relacionada ao ensino.

Após a elaboração do perfil proposto, verificou-se também a necessidade de se avaliar futuramente as publicações em periódicos provenientes dos estudos analisados, constatando se há ou não a difusão das informações dessas pesquisas, de forma que as mesmas não fiquem restritas no âmbito nacional, proporcionando, assim, maiores avanços para a enfermagem.

\section{REFERÊNCIAS}

1. Lopes ACC, Ferreira AA, Fernandes JAL, Morita ABPS, Poveda VB, Souza AJS. Construction and evaluation of educational software on urinary indwelling catheters. Rev Esc Enferm USP [serial on the Internet]. 2011 [Cited 2012 July 12]; 45(1): 21522. Available from: http://www.scielo.br/pdf/reeusp/v45n1/30.pdf

2. Fonseca LMM, Góes FSN, Ferecini GM, Leite AM, Mello D, Scochi CGS. Inovação Tecnológica no ensino da Semiotécnica e Semiologia em enfermagem neonatal: do desenvolvimento à utilização de um software educacional. Texto Contexto Enferm 
Florianópolis [serial on the Internet]. 2009 July-Sept [Cited 2012 July 10]; 18(3): 54958. Available from: http://www.scielo.br/pdf/tce/v18n3/a19v18n3.pdf

3. Baptista PCP, Felli VEA, Mininel VA, Karino ME, Silva SM, Tito RS, et al. Using technological innovation as a tool to monitor nursing workers health. Rev Esc Enferm USP [serial on the Internet]. 2011 Dec [Cited 2012 July 11]; 45(esp): 1621-1626. Available from: http://www.scielo.br/pdf/reeusp/v45nspe/v45nspea13.pdf

4. Dalri MCB; Carvalho EC. Planejamento da assistência de enfermagem a pacientes portadores de queimadura utilizando um software: aplicação em quatro pacientes. Rev. Latino-am. Enfermagem [serial on the Internet]. 2002 Nov-Dec [Cited 2012 July 11]; 10(6): 787-793. Available from: http://www.scielo.br/pdf/rlae/v10n6/v10n6a6.pdf

5. Rodríguez EOL, Guanilo MElE, Fernandes LM, Candundo G. Informática em enfermagem: facilitador na comunicação e apoio para a prática. Invest Educ Enferm [serial on the Internet]. 2008 Sept [Cited 2012 July 10]; 26(2 supl): 144-149. Available from: http://aprendeenlinea.udea.edu.co/revistas/index.php/iee/article/view/2871/2396

6. Santos SR. Computers in nursing: development of free software application with care and management. Rev Esc Enferm USP [serial on the Internet]. 2010 [Cited 2012 July 11]; 44(2): 295-301. Available from: http://www.scielo.br/pdf/reeusp/v44n2/08.pdf

7. Lopes EM, Pinheiro AKB, Pinheiro PNC, Vieira NFC. Technology and nursing practice - a bibliographical research. Online Braz J Nurs [serial on the Internet]. 2009 [Cited 2012 July 12]; 8(1). Available from: http://www.uff.br/objnursing/index.php/nursing/article/view/j.1676$\underline{4285.2009 .1883 / 446}$

8. Ludke M, André MED. A pesquisa em educação: abordagens qualitativas. São Paulo: EPU; 1986.

9. Gil AC. Como elaborar projetos de pesquisa. $4^{\mathrm{a}}$ ed. São Paulo: Atlas; 2007.

10. Marconi MA, Lakatos EM. Fundamentos da metodologia científica. $6^{\mathrm{a}}$ ed. São Paulo: Atlas, 2009.

11. CAPES [internet]. Brasília: Coordenação de Aperfeiçoamento de Pessoal de Nível Superior; 2009 Nov 06. [Cited 2012 Jun 12]. Available from: http://capes.gov.br/servicos/sala-de-imprensa/36-noticias/3316-banco-de-teses-dacapes-possui-mais-de-450-mil-resumos

12. Leopardi MT. Metodologia da pesquisa em saúde. $2^{\mathrm{a}}$ ed. Florianópolis: UFSC/PósGraduação em Enfermagem, 2002.

13. Rocha Neto. Prospectiva da Pós-Graduação no Brasil (2008 - 2022). RBPG [serial on the Internet]. 2010 July [Cited 2012 July 12]; 7(12): 58-79. Available from: http://www2.capes.gov.br/rbpg/images/stories/downloads/RBPG/Vol.7_12/3_ARTIG $\underline{\text { O.pdf }}$

14. Goldani MZ, Silva CH, Nascimento LFM, Blank D. A questão da produção do conhecimento: desafios na gestão dos programas de pós-graduação. RBPG [serial on the Internet]. 2010 July [Cited 2012 July 11]; 7(12): 104-116. Available from: http://www2.capes.gov.br/rbpg/images/stories/downloads/RBPG/Vol.7_12/5_ARTIG $\underline{\text { O.pdf }}$

15. Schwartzman S. A transição necessária da pós-graduação brasileira. Jornal da Ciência On-line [serial on the Internet] 2010 Apr [Cited 2012 July 11]. Available from: http://www.schwartzman.org.br/simon/capes2010.pdf

16. Brasil. Ministério da Saúde. Secretaria de Ciência, Tecnologia e Insumos Estratégicos. Departamento de Ciência e Tecnologia. Política nacional de ciência, tecnologia e inovação em saúde. $2^{\mathrm{a}}$ ed. Brasília: Editora do Ministério da Saúde, 2008. p. 44.

17. Aquino PS, Melo RP, Lopes MVO, Pinheiro AKB. Analysis of the concept of technology in nursing according to the evolutionary method. Acta Paul Enferm [serial 
on the Internet]. 2010 Sept-Oct [Cited 2012 July 11]; 23(5): 690-6. Available from: http://www.scielo.br/pdf/ape/v23n5/17.pdf

18. Zem-Mascarenhas SH, Cassiani SHB. Desenvolvimento e avaliação de um software educacional para o ensino de enfermagem pediátrica. Rev Latino-am Enfermagem [serial on the Internet]. 2001 Nov-Dec [Cited 2012 July 11]; 9(6): 13-18. Available

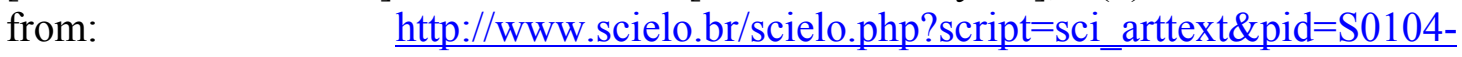
$11692001000600003 \& \operatorname{lng}=\mathrm{pt \& nrm}=$ iso

19. Mendes IAC; Trevizan, MA, Évora YDM. Comunicação e enfermagem: tendências e desafios para o próximo milênio. Esc. Anna Nery Rev. de Enferm [serial on the Internet]. 2000 Ago [acesso em 2012 jul 11]; 4 (2): 217-224. Available from: http://redalyc.uaemex.mx/redalyc/pdf/1277/127718323009.pdf

20. Bellini C, Garcia MH, Marziale MHP. Utilização de recurso tecnológico como agente facilitador do trabalho de enfermagem. Rev Latino-am. Enfermagem [serial on the Internet]. 1996 July [Cited 2012 July 11]; 4(2): 101-111. Available from: http://www.scielo.br/scielo.php?script=sci arttext\&pid=S0104$11691996000200008 \& \operatorname{lng}=$ es\&nrm=iso

21. Mendes IAC, Leite JL, Trevizan MA, Trezza MCSF, Santos RM. A produção tecnológica e a interface com a enfermagem. Rev. Bras. Enf [serial on the Internet]. 2002 Sept-Oct [Cited 2012 July 11]; 55(5): 556-561. Available from: http://gepecopen.eerp.usp.br/files/artigos/Artigo121fin.pdf 